\title{
Anemia ferropriva y respuesta inmune celular
}

\author{
Liana Schlesinger F. ${ }^{1}$; Manuel Olivares G.2. \\ Marianela Arévalo N. ${ }^{3}$; Patricia Chadud M. ${ }^{4}$
}

\section{Iron deficiency anemia and cell-mediated immunity}

The available information on the effecls of iron deficiency on immune response is contradictcry. The presence of inleclions, melnutrilion, dillerences of age belween polienls and conlrols, and deficiences of nutrients other than iron complicates tine interpretaticn of immundogical results. Fifteen iron deficient anemic and eleven conlrol infants witheut iron deficicacy, all aged 15 to 19 months and wilncut another nutrifional deprivalion neither infections were stucied to define the ettecis of iron deticiency anemia on cellular :mmune response. Delayed culaneous hypersensitivity reactions to 2. 4 dinitrochlorobenzene and PPD and lymphopicliferolive response to phyohemaglutinin were measurec in beth palients orid conirols. No significani dillerences in the immune response were observed between boh groups before and ctrer a three months Itealment with $5 \mathrm{mg} \cdot \mathrm{kg}$ - day elcmenial iron given as ferrous sulfale. These results suggest that mild iton deficiency anemia would not impair cellular immune response.

Key wards: iron deficiency, anernia, cellular imrnunify, delayed hyperensitivily, infants.i

La deficiencia de hierro es una de las carencias nutricionales más prevalentes y la causa principal de ancmia en la infancia ${ }^{1}$. El lactante, por su gran velocidad de crecimiento y el bajo aporte de hierro en su dieta, constituye uno de los grupos de edad cn que la deficiencia de hierro es muy prevalente. En nuestro país $28 \%$ de los niños menores de 2 años sufre de anemia y más del doble ticnen manifestaciones bioquímicas de deficiencia de hierro ${ }^{2}$.

Entre las expresiones no hematológicas de deficiencia de hierro se mencionan alteraciones de la respuesta inmunc, como rcducción de la capacidad bactericida de los neutrólilos ${ }^{3,4}$ y del número o proporción de linfocitos $\mathrm{T}^{5-9}$. Diversos esludios se han enfocado en el efecto de la deficicncia de hierro sobre la inmunidad celular, con resultados contradictorios ${ }^{5,10-16}$.

Con el propósito de dilucidar si la deficiencia de hicro afecta la respuesta inmune celular se determinó la respuesta proliferativa de los

1. Unidad de Innunología, NTA, Universidad de Chile.

2. Unidad de Hematología, INTA, Universidad de Chile

3. l'cenólogo Médico. Unidad de Inmunología, INTA, Universidad de Chile.

4. Tecnólogo Médico. Unidad de Ilematologja, INTA, Universidad de Chile. linfocitos y la reacción de hipersensibilidad retardada en un grupo bien caracterizado de laclantes con ancmia ferropriva y en niños controles sin deficiencia de hierro, antes y despućs de suplementar su alimentación con hierro medicinal.

\section{Personas y métodos}

Se deleminó lá respuesta inmunitaria cclular en 15 lactantes con anemia feropriva, cuyas edades fluctuaron en tre 15 y 19 meses. Once lactantes de edades y condiciones comparables sirvicton como grupo control. Los niños selec. cionados proven Ĺat1 de un estudio de tereno de prevención de deficiencia de hierro con ieches fortificadas. Todos los laclantes eran cutróficos, su peso de nacimiento había sido superior a $2500 \mathrm{~g}$ y no prescrtaban infecciones en el mo. mento del estudio. Ninguno de ellos había recibido vacunas vivas atenladas en los tres meses previos al estudio. Los cnterios utilizados en la definición de anemia por deficien. cia de hierru fueron: concentracion de hemoglobina (Hb) $\leq$ $10,5 \mathrm{~g} / \mathrm{dl}$ junto con saturación de transferrina (Sar) $<9 \%$, proluporlirina Jibre critrocilaria $(\mathrm{PLE})>100 \mathrm{ug} / \mathrm{dl}$ de glóbulos rojos (GR), ferrilina sérica (FS) $<10 \mathrm{ug} / \mathrm{y}$ aumento del nivel de $\mathrm{Hb}$ superior a $1 \mathrm{~g} / \mathrm{dl}$ (prueba lerapéutica positiva) al cabo de tres meses de tratamiento con $5 \mathrm{mg} \cdot \mathrm{kg}$ - día de hicrro elemental en forma de sulfato fcrroso oral. Los laciantes control tenían todos Jos paránetros indicadores de la nulrición de hierro deniro de márgenes normales y prueba terapética ncgativa.

Nutrición de hierro y folatos: en todos los pacientes se midieron concentraciones de $\mathrm{Hb}^{17}$, hierro sérico, capacidad 
de combinación de hierro y $\mathrm{Sat}^{18}, \mathrm{PLE}^{19}, \mathrm{FS}^{20}$, y concentraciôn de folatos séricos y eritrocitarios 21,22 .

Reacciones cutáneas de hipersensibilidad retardada: pa ra el estudio de sensibilización con 2-4 dinitroclorobernceno (DNCB) se aplicé $0,1 \mathrm{~m}$ de unna solución al $2 \%$ de DNCB en acetona en un área de $1 \mathrm{~cm}$ de diámetro del antebrazo. Veinte días después se aplićs $0.1 \mathrm{ml}$ de una solución de DNCB al $0,1 \%$ en el antebrazo opuesto y se leyo la reacción a las $4 B$ horas ${ }^{23}$. Se considers como reacción positiva la presencia de induración y, o, vesiculación. La prueba cutánea de sensibilidad al PPD se realizó inyectando intradérmicamente $0.1 \mathrm{ml}$ de una solución de uberculina de $2 \mathrm{LI}$. pues todos los nirios tenían cicatrix de inmunización con BCG. Se consideró como reacción positiva la presencia de una induración $\geq 5 \mathrm{~mm}$ de diámetro a las 48 horas ${ }^{23}$.

La determinación de la capacidad linfoproliferativa frente a fitchemaglutinina se realizó separando linfocitos de sangre pcriférica mediante gradiente de Ficoll Hypaque. Sie cultivaron $1 \times 10^{5}$ linfocitos en presencia de RPMI 1640, cun $20 \%$ de suero autólogo, anubióticos, giutamina y fíchemaglutinina (PHA), durante 96 horas, en atmósfera húmeda en una mezcla ambicntal de $5 \%$ de $\mathrm{CO}_{2}$ y $95 \%$ de aire ${ }^{24}$. Todos lus euluivos se realizaron en triplicados y como controles se realizaron cultivos sin PHA. Dieciocho horas antes del têrminu del período de incubación se agregó $0,5 \mu \mathrm{Ci}$ de timidina tritiada, actividad específica 6,7 $\mathrm{kmol} / \mathrm{mCi}$ (New Emgland Vuclear, Boston Mass. USA) a cada cultivo. Al término del período de incubación todos lus culcivos sc cosecharon en un recolector automático de células. I id incorporación de timidina tritiada se midió en un contador de centelleo líqui. do. Los resultados se expresaron como cuentas por minutos (cpm) nelas, es decir, cpm de culivos estimuladus com $\mathrm{PHA}$ menos cpm de cullivos sin cstimular.

La cvaluación del estado de nutrición de hierro y el estudio inmunológico se realizaton antes y después de 3 meses de tratamicnto con hicrro elemental $5 \mathrm{mg} \cdot \mathbf{k g} \cdot$ día, cn forma de gotas de sulfato ferroso. Para realizar el estudio se solicitó a los padres su consentimiento informado $y$ se obtuvo la aprobación del Conité de Etica de] Instisuto de Nutrición y Tecnología de los Alimentos.
I.a evaluación estadística se realizó mediante pruebas paramétricas como la de $t$ de Student pareada y no pareada, ensayos no paramétricos como Mann Whitney, Friedman y prueba exacta de probabilidad de Fisher.

\section{Resultados}

Nutrición de hierro y folato: en la tabla 1 se mucstran los indicadores de nutrición de hicro en pacientes y controles. Se observa que los pacientes presentan una anemia ferropriva leve, la que se recupera después de 3 meses de tratumiento con hierro medicamentoso. El grupo control no presenta signos de deficiencia de hierro y sus indices hematológicos no se modifican después del tratamiento con sulfato ferroso. Los niveles de folato sérico y eritrocitario se encontraron sobre $3 \mathrm{ug} / 1$ y $160 \mathrm{ug} / \mathrm{l}$ eritrocilos respectivamente, al inicio y al término del estudio en todos los lactantes.

Respuesta inmune: En 93,3\% de los casos, los lactantes con anemia fueron capaces de sensibilizarse al 2-4 DNCB (tabla 2). Sin embargo, un porcentaje significativamente menor de lactantes anémicos presentó respuesta cutánea positiva al PPD en comparación al grupo control $(p<0,03$, t de Student (tabla 2). Después del tratamiento con sulfato ferroso se repitió la intradermorrcacción de PPD en los 6 niños anémicos cuya reacción cutánea era negativa antes del tratamiento, tornándose ésta positiva en sólo uno de ellos.

No se observaron diferencias estadísticamente significativas en la capacidad proliferativa de

Tabla 1

Incticadores de laberatorio de nutrición de hisro en lactantes anémicos y controles antes y después del tratamiento con sulfato ferroso

\begin{tabular}{|c|c|c|c|c|}
\hline & $\begin{array}{l}\text { Anémicus } \\
\text { Pre-irat. } \\
\text { (n: 15) }\end{array}$ & $\begin{array}{c}\text { Anemicos } \\
\text { Post-trat. } \\
\text { (n: 15) }\end{array}$ & $\begin{array}{c}\text { Cuntroles } \\
\text { Pre-(rat. } \\
\text { (n: 11) }\end{array}$ & $\begin{array}{c}\text { Contrules } \\
\text { Post-trat. } \\
\text { (n: } 11)\end{array}$ \\
\hline $\mathrm{Hg}(\mathrm{g} / \mathrm{dl})$ & $9.8 \pm 0,6$ & $12,9 \pm 1,01 \mathrm{a}$ & $13,3 \pm 0,8 b$ & $13,1 \pm 0,6$ \\
\hline $\operatorname{Sat}(\%)^{*}$ & $5,2 \pm 1,3$ & $19,2 \pm 12,00 \mathrm{a}$ & $21,2 \pm 5,7 b$ & $34,5 \pm 42,1$ \\
\hline PLF (ug/dl GR)** & $183,0 \pm 62,0$ & $119,0 \pm 19,00 a$ & $75,0 \pm 13,0 b$ & $62,0 \pm 14,0$ \\
\hline Ferritina $(\mathrm{ag} f)^{* * *}$ & $9(5-17)$ & $32(20-53) a$ & $34(23-50) \mathrm{b}$ & $31(22-43)$ \\
\hline
\end{tabular}

Resultados expresados como $x \pm D E$.

* Saturación de la transferrina

** Pfotoporfirina libre eritrocilaria

* * Ptomedio geométrico y rango de $1 \mathrm{DE}$

a: $p<0,01$ (i pareado) en relación a grupo anémico pre-tratamiento

b: $p<0,01$ ( $t$ no pareado) en relación a grupo anérnico anles del trataniento 
Tabla 2

Respuesta cutánea de hipersensibilidad retardada en lactantes con anemia ferropriva*

\begin{tabular}{lccc} 
& Anémicos & Controles & $\mathbf{p}$ \\
\hline PPD ** & $7 / 14$ & $10 / 11$ & $<0,05$ \\
DNCB & $14 / 15$ & $11 / 11$ & ns
\end{tabular}

* Resultados se expresaron como número de rcaccioncs positivas con respecto al total.

** Todos los niños aralizados presentaban cicatriz BCG.

linfocitos frente a PHA entre lactantes anémicos y controles antes del tratamiento con hicrro (tabla 3). Sin embargo, en los niños que sufrían anemia, la capacidad linfoproliferativa aumentó al cabo de tres meses de tratamicnto con sulfato ferroso, si bien la diferencia no alcanzó a ser estadísticamente significativa.

\section{Comentario}

Este estudio muestra que no existe alteración de la respuesta inmunitaria celular en la anemia por deficiencia de hierro, cuando se la determina por la respuesta de hipersensibilidad retardada y la capacidad linfoproliferativa. Entre los numerosos trabajos que han investigado el efecto de esta deficiencia sobre la respuesta proliferativa de los linfocitos en respuesta a mitógenos y antígenos se han obtenido resultados discordantes, pues en algunos ha sido normal ${ }^{25-29}$, mientras en otros estaba deprimida ${ }^{5,10-14}$, comunicándose en en este último caso algunas experiencias en que la alteración era corregible con la administración de hierro ${ }^{5,11}, 12,14$. Cabe reiterar que muchos de estos estudios presentan limitaciones metodológicas o fueron rcalizados en pacientes de diferentes grupos de edad, lo cual dificulta la interpretación de los resultados inmunológicos. Por estas razones este análisis se hizo en un grupo bien caracterizado de lactantes sin antecedentes de peso bajo al nacer, infección, malnutrición o deficiencia de ácido fólico, en los cuales se evaluó adecuadamente la nutrición de hierro. La respuesta linfoproliferativa en los lactantes anémicos y controles bien nutridos en hierro antes y después de suministrar suplementos con hierro medicinal estaba dentro de los límites aceptados como normales y aumentó, aunque no de manera
Tabla 3

Reactividad linfocitaria frente a PHA en lactantes con anemia ferropriva antes y después del tratamiento con sulfato ferroso

\begin{tabular}{|c|c|c|}
\hline & \multicolumn{2}{|c|}{$\begin{array}{l}\text { Incorporación de timidina } \\
\text { tritlad a (cpm x } 105 \text { linfocitos) }\end{array}$} \\
\hline & $\begin{array}{c}\text { Anérnicos } \\
(\mathrm{n}: 15)\end{array}$ & $\begin{array}{c}\text { Controles } \\
(\mathrm{n}: 11)\end{array}$ \\
\hline $\begin{array}{l}\text { Pre-tratamiento } \\
\text { Post-tratamiento } \\
\text { P }\end{array}$ & $\begin{array}{c}39737 \pm 100 \\
62823 \pm 169 \\
\text { ns }\end{array}$ & $\begin{array}{c}60558 \pm 413 \\
42781 \pm 176 \\
\text { ns }\end{array}$ \\
\hline
\end{tabular}

Resultados expresados como $\mathrm{X} \pm \mathrm{ESM}$

significativa, en los niños con anemia. Estos resultados concuerdan con otros realizados en ninos $^{27-28}$ y adultos ${ }^{26-29}$. Existe información que sugiere que alteración de Ja inmunidad celular sería debida a menor actividad de la nucleótido ribonucleasa, enzima indispensable para la sintesis del DNA, la que para su adecuada función requiere de un aporte continuo de hierro ${ }^{30}$.

La deficiencia de hierro no alteró, en esta serie, la capacidad de los lactantes a sensibilizarse con DNCB, hecho que indicaria que no existiría una altcración en el reconocimiento y procesamiento antigénico. Este hallazgo es concordante con observaciones en que $100 \%$ de un grupo de adultos deficientes en hierro fueron capaces de responder al $\mathrm{DNCB}$, mientras en la deficiencia de ácido fólico sólo el $4 \%$ tuvo una reacción cutánea positiva ${ }^{26}$.

La respuesta cutánea significativamente menor de estos lactantes con anemia al PPD persistió después del tratamiento co hierro, lo que hace poco probable que sea causada por la carencia de este nutriente. Existe escasa información acerca del efecto de la deficiencia de hierro sobre la producciốn de linfoquinas, pero se ha demostrado menor producción de facror inhibidor de la migración de macrófagos (MIF) en sujetos adultos con anemia ferropriva ${ }^{10}$, los que se recuperaron con terapia marcial ${ }^{31}$. Por otra parte, en ratas con anemia ferropriva severa se observó disminución de la producción de $\mathrm{IL}_{-1} \mathbf{3}^{32}$, en cambio en lactantes con anemia ferropriva la producción y liberación de la misma interleuquina era nor$\mathrm{mal}^{33}$. Cabe señalar que en la detección de IL-1 en lactantes se utilizó un inmunoensayo específico, en cambio en los animales la determinación 
se realiz6 mediante un bioensayo. Recientemente se ha descrito tambiến menor producción de interleuquina-2 en niños anémicos ferroprivos ${ }^{34}$, sin embargo llama la atención que en cse mismo estudio los autores no encontraron disminución de la capacidad proliferativa de los linfocitos.

La ausencia de alteraciones causadas por la anemia por deficiencia de hierro en la respuesta inmunitaria celular se sustentaría además en que no ha detectado en estos pacientes mayor frecuencia de infecciones por Pneumocystis carini, infecciones virales, fúngicas sistémicas, propias de las inmunodeficiencias celulares. Aún más, en tres estudios de terreno prospectivos realizados en lactantes de la ciudad de Santiago no se demostraron diferencias en la incidencia de diarrea o infecciones respiratorias entre los sujetos que recibieron alimentos fortificados con hierro y aquellos que no lo hicieron, a pesar que entre estos últimos la prevalencia de anemia fue cercana a treinta por ciento ${ }^{\mathbf{3 5 - 3 7}}$.

Basados en nuestros resultados podemos afirmar que la anemia ferropriva leve no alteraría la respuesta inmune celular, sin embargo no podemos descartar que anemias de mayor severidad pudieran determinar una depresión de la función inmunológica.

\section{Resumen}

Existe informacion contradictoria en relación al efecto de la carencia de hicrro sobre respuesta inmune. La interpretación de los datos inmunológicos obtenidos en pacientes con anemia ferropriva varía según edad, estado nutricional, presencia de infecciones y asociación con otras deficiencias nutricionales. Para definir el efecto que ejerce la anemia ferropriva sobre la respuesta inmunitaria celular se seleccionaron 15 lactantes con anemia ferropriva y 11 controles adecuadamente nutridos con hierro, entre 15 y 19 meses de edad, eutróficos, sin infecciones ni deficiencias nutricionales asociadas. Se determinó la capacidad de sensibilización a antígenos que evocan respuestas cutáneas de hipersensibilización retardada con 2 - 4 dinitroclorobenceno y PPD, como también la capacidad proliferativa de sus linfocitos al estímulo con fitohemaglutinina. No se observaron diferencias significativas en la respuesta inmune celular entre pacientes y controles antes y al cabo de tres meses de tratamiento con
$5 \mathrm{mg}$ - $\mathrm{kg}$ - día de hierro elemental en forma de sulfato ferroso. Se sugiere que la anemia ferropriva leve no alteraría la respuesta inmune celular.

(Palabras clave: hierro, deficiencia, anemia, inmunidad celular, hipersensibilidad retardada, lactantes.)

\section{Referencias}

1. Florentino RFR, Guirriec RM: Prevalence of nutritional anemias in infancy and Childhood with emphasis on developing countries. En: Stekel A, ed. Iron Nutrition in Infancy and Childhood. New York: Raven Press $1984 ; 61-74$.

2. Ríos $E_{1}$ Otivares $M$, Amer $M$, Chadud $P$, Stekel A. Evaluation of iron status and prevalence of iron deficiency in infants in Chile. En: Underwood BA, ed, Nutrition Intervention Strategies in National Development. New York: Raven Press 1983; 273-283.

3. Chandra $R K$ : Reduced bactericidal capacity of polymorphs in iron deficiency. Arch Dis Child 1973; 48: 864-866.

4. Waiter $T$, Arredondo S, Arévaio M, Siekel A: A effect of iron the rapy on phagocylosis and barericidal activity in neutrophils of ison-deficient infants. Am J Clin Nutr 1986; 44:877-882.

5. Chandra $R K$ : Impaired immunocompetence associaled with iron deficiency. J Pedialr 1975; 86: 899-902.

6. Skrikantia SG, Bhaskaram C. Prasad JS, Krishramachari $K A V R$ : Anaemia and immune response. Lancel 1976; II: 1307.1309

7. Bagehi K. Mohanram $M$, Reddy $V$ : Humoral immune response in children with iron-deficiency anaemia. Bril Med J. 1980; 1249-1251.

8. Prema $X$, Ramaiakshmi BA, Madhavadepeddi $R, B a b u$ $S$ : Inmune status of anaemic pregnant women. Brit $\mathbf{J}$ Obstet Gynaecol 1982; 89: 222-225.

9. Berger $J$ Schneider D, Dyck JL, Aplogan AJA, Galan $P$, fercberg $S$ : Iron deficiency, cell-mediated immunity and infection among 6.36 months old children living in rural Togo. Nutr Res 1992; 12: 39-49.

10. Joynson DHM, Waiker M, Jacobs A, Dolby AE: Defect of cell-medialed immunity in patients with iron-deficiency anaemia. Lancel 1972; II: 1058-1059.

11. Macdougall LG, Anderson R, MacNab GM, Katz J: The immune response in iron-deficient children: impaired cellular defense mechanisms with altered humoral components. J Pediatr 1975; 6: 833-843.

12. Fletcher $J$, Mather J, Lewis MJ, Whiting G: Mouth lesions in iron deficient anemia: Relationship to Candida albicans in saliva and to impairment of lymphocyte transformation. J Infect Dis 1975; 131 : 44-50.

13. Sawisky B, Kanier $R$, Sawitsky A: Lymphocyte response to phytomitogens in iron deficiency. Am J Med Sci 1976: 272: 153-160.

14. Krantman HJ, Young SR, Ank BJ, ODonned MC. Rachelefsky GS, Stiehman RE: Inmune function in pure iron deficiency. Am $J$ Dis Child 1982; 136: 840 B44. 
15. Daliman PR: Jron deficicncy and the immune responsc. Am J Clin Nulr 1987: 46: 329-334.

16. Dhur A, Golan P, Hercberg $S$ : Iron status, immune capacity and resistence to infections. Comp Biochern Physiol 1989; 94A: 11-19.

17. International Commiltee for Siandarzitation in Haemotology. Kecommendation requirements for hacmoglobinometry in human bood. Clin Pathol 1965; 18 : 353-355.

18. Fischer DS, Price DC: A simple serum iron method using the new sensitive chromogen tripyridyl-s-triazine. Clin Chem 1964; 10: 21-30.

19. Heller $S R$, Labbe $R F, N$ utter $J A: A$ simplified assay for porphyrins in whole blood. Clin Chem 1971; 17:525528.

20. Gomma Dab (125-I) Ferritin Radioimmunoassay Kit Clinical assay, Division of Travenol Laboratories Inc. Cambridge, Mass, USA.

21. Waters $A J$, Mollin DL: Sudies on the folic acrivity of human serum. J Clin Pathol 1961; 14: 335-344

22. Hoffrand $A V$, Beverly $F A$, Mollin $D L$. Method of assay of red cell folate activity and the value of assays as a test for fo] atc de[iciency. J Clin Pachol 1966; 19: 17-28.

23. Worls Weath Organization Scientific Group: Inmunodeficiency tcsis for assessing immune stalus. WHO Tech Rep Ser 1978; 630: 1.98.

24. Oppenheim $J J$, Rosentreich $D L$ : Lymphosytc transformation: Ulilizistion of automatic cell harvester. In: Bloom BR, David JR, eds. In vitro methods in cell-mediated Iumor Immunity. New York: Acadenic Press 1976: $73-85$

25. Kulapongs $P$, Suskind $R$, Vithayasi $V$, Olsen RE: Cellmediated immunity and phagocylosis and killing function in children with severe iron-deficiency anacmia. Lancel 1974; II: 680-691.

26. Gross RL, Reid NO. Newberne PM, Aurgess B, Marsion $B A R, H i f t W:$ Depressed cell-mediated immunity in megaloblastic anemia due 10 folic acid deficiency. Am J Clin Nutr 1975; $28: 225-232$
27. Gupla KK, Dhan PS, Singh $H$ : Cell-mediated immunity in children with iron-deficjency anaemia. Indian J Pediatr 1982; 49:507.510.

28. Grosch-Wörner I, Grosse-Widde H, Bender-Göre Ch, Schäfer $K H$ : Lymphozytcnfunktionen bei Kindem mit Eisenmangel. Klin W 1984; 62: 1091-1093.

29. Moraes de Souza H, Kerbauy J, Yamamoto M, Da Silva MP. Dos Santos MRM: Depressed cell-mediated immunity in iron defiency anemia duc to chronic loss of blood. Braz J Med Biol Res 1984; 17: 143-150.

30. Thelander L, Gräslund A. Thelander $M$ : Continual presence of oxygen and iron required for mammalian ribonucleotide reduction: possible regulation mechanism. Biochem Biphys Res Commun 1933; 110; 859 865

31. Jacobs A, Hoynson DHM: Lymphocyte funclion and iron defieiency anacmia. Lancel I974; II: 844.

32. Helyar $L, 5$ herman $A R$ : Iron deficiency and interleukin production by rass leukocytes. Am J Clin Nutr 1987; 46: 346-352.

33. Muñoz C, Olivares $M$, Lopez $M$, Simon $V$, Letelier A. Arevalo $M$, Schlesinger $L$ : Effect of isolated jon deficiency in monocyte-derived cylokine production in infants. J Cell Biochem 1993; suppl 17B:95.

34. Galan P. Thibault H, Preziosi P, Mercberg $S$ : Interleukin 2 production in iron-deficient children. Biol Trace Elem Res 1992; 32: 421-426.

35. Heresi $G$, Orivares $M$. Pizarno F, Cayazzo $M$, Stekel $A$ : Effect of an iron fortified milk on mobidity in infancy. A field trial. Nutr Res 1987; 7: 915-922.

36. Olivares $M$, Pizarro F, Ileresi $G$, Hertrampf E, Cayazzo $M$, Wailer $T$ : Effecl of a hemoglobin forified cereal on morbidity in breast fed infants. En: Recent knowledge on iron and folate deficiencies in the world. Hercberg S, Gatan P, Dupin H, eds. Colloque INSLRM París 1990; 197: 653-658.

37. Walier T, Olivares M. Pizarro F: Iron and infection. En: Dictary iron: birth to two years. Filer $[\mathrm{J} J \mathrm{r}$, ed. Raven Press: New York, 1989: 119-139. 